\author{
Barbara Horejs \\ mit Beiträgen von
}

Alfred Galik und Ursula Thanheiser

\title{
Erster Grabungsbericht zu den Kampagnen 2006 und 2007 am Çukuriçi Höyük bei Ephesos*
}

\begin{abstract}
Einleitung
Wie in so vielen antiken Fundorten an der mittleren Westküste der Türkei lag der Fokus der Forschungen aufgrund der Größe, Bedeutung und oberflächlich erhaltenen Ruinen auch in Ephesos lange Zeit auf den antiken und spätantiken Perioden ${ }^{1}$. Mit den ersten prähistorischen Großprojekten der letzten beiden Jahrzehnte an der westanatolischen Küste rückt nun auch dieser Raum vermehrt in den Blickpunkt vorgeschichtlicher Forschungsinteressen ${ }^{2}$. Die Notwendigkeit dazu wird vor dem Hintergrund überregionaler Fragestellungen vom Neolithikum bis in die Spätbronzezeit mehr als deutlich ${ }^{3}$. Während Zentral-, Süd- und Ostanatolien ebenso wie der gesamte ägäische Raum und Südosteuropa zum Kerngebiet prähistorischer Archäologie zählen, lag Westanatolien zumeist außerhalb der wissenschaftlichen Schwerpunktforschung.

Dieses Forschungsdesiderat veranlasste den damaligen Grabungsleiter von Ephesos, F. Krinzinger, ein Grundlagenforschungsprojekt zur Prähistorie nun auch in der Mikroregion von Ephesos zu initiieren und zu unterstützen ${ }^{4}$. Nach ersten Begehungen der Umgebung von Ephesos im Herbst 2005 fiel die Entscheidung, den Fokus der Forschungen zunächst auf einen Tell südöstlich der antiken Stadt zu richten (Abb. 1), der bereits als Çukuriçi Höyük in die Literatur eingeführt worden war ${ }^{5}$. Folgende Faktoren waren für diese Wahl schließlich ausschlaggebend:

- Der Hügel war 2005 noch in einer Höhe bis zu 4,5 m über dem heutigen Laufhorizont erhalten ${ }^{6}$, was mehrere Kulturschichten erhoffen ließ. Mit einer flächigen Gesamtausdehnung von rund $100 \times 80 \mathrm{~m}$ waren des Weiteren Reste von Siedlungsstrukturen zu erwarten (Abb. 2).

* Für das Gelingen der ersten beiden Grabungen sei vielen Personen gedankt, allen voran dem Initiator F. Krinzinger, allen Kooperationspartnern (F. Pirson, E. Pernicka, B. Weninger, M. Mehofer, A. Galik, U. Thanheiser), Ch. Kurtze (Geodäsie), N. Gail (Photographie) sowie den studentischen Mitarbeiter/-innen und türkischen Grabungsarbeitern. - Zahlreiche Literaturhinweise, die Einsicht in unpubliziertes Fundmaterial sowie fruchtbare Diskussionen verdanke ich N. Karul, M. Özdoğan, T. Efe, H. Erkanal, J. Maran, O. Kouka, V. Şahoğlu, C. Çilingiroğlu, J. Seeher, U.-D. Schoop, R. Krauß, A. Reingruber, E. Alram-Stern und P. Pavúk. - Zitate und Abkürzungen folgen den Richtlinien des Österreichischen Archäologischen Instituts <http://ww.oeai.at/ publik/autoren.html>, bibliographische Angaben zu den zusätzlich verwendeten Kurzzitaten finden sich am Ende des Beitrags.

${ }^{1}$ Exemplarisch mit weiterführender Literatur s. Friesinger - Krinzinger 1999; Scherrer 2001; Groh 2006.

${ }^{2}$ Beispielsweise das Großprojekt IRERP in der Region von Izmir mit Schwerpunktforschungen in Liman Tepe, Panaz Tepe und Baklatepe unter den Leitungen von A. und H. Erkanal (Informationen und Literatur s. <http://www.geocities.com/irerp_tr/>); Milet (Niemeier 2007 mit älterer Lit.); Metropolis - Bademgediği Tepe (Meriç - Mountjoy 2002; Meriç 2003; Meriç 2007); Ulucak (Çilingiroğlu u. a. 2004); jüngst auch auf der Insel Tavşan Adası bei Didyma (DAI Jahresbericht 2006).

${ }^{3}$ Exemplarisch s. die Diskussion ausgewählter Fragenkomplexe vom Neolithikum bis zur Bronzezeit bei Özdoğan - Başgelen 1999; Hauptmann - Özdoğan 2007; Özdoğan 2007; Lichter 2005; Parzinger 1993; Schoop 2005; Cutting 2005; Yakar 1985; French 1997; Kouka 2002; Lloyd - Mellart 1962; Niemeier 2007; zur Spätbronzezeit in Ephesos s. hier B. Horejs, Eine spätbronzezeitliche Bestattung in Halkapınar bei Ephesos, ÖJh 77, 2008, 107-129.

${ }^{4}$ An dieser Stelle möchte ich mich bei F. Krinzinger herzlich für die konsequente Unterstützung sowohl in Ephesos als auch in Wien bedanken, die dieses Projekt erst ermöglicht hat.

${ }^{5}$ Evren - İçten 1998; Evren 1999; Çevik 2002, 72 f.

${ }^{6}$ Für eine dreidimensionale Ansicht in Form eines Modells s. Horejs 2008, Abb. 4.
\end{abstract}




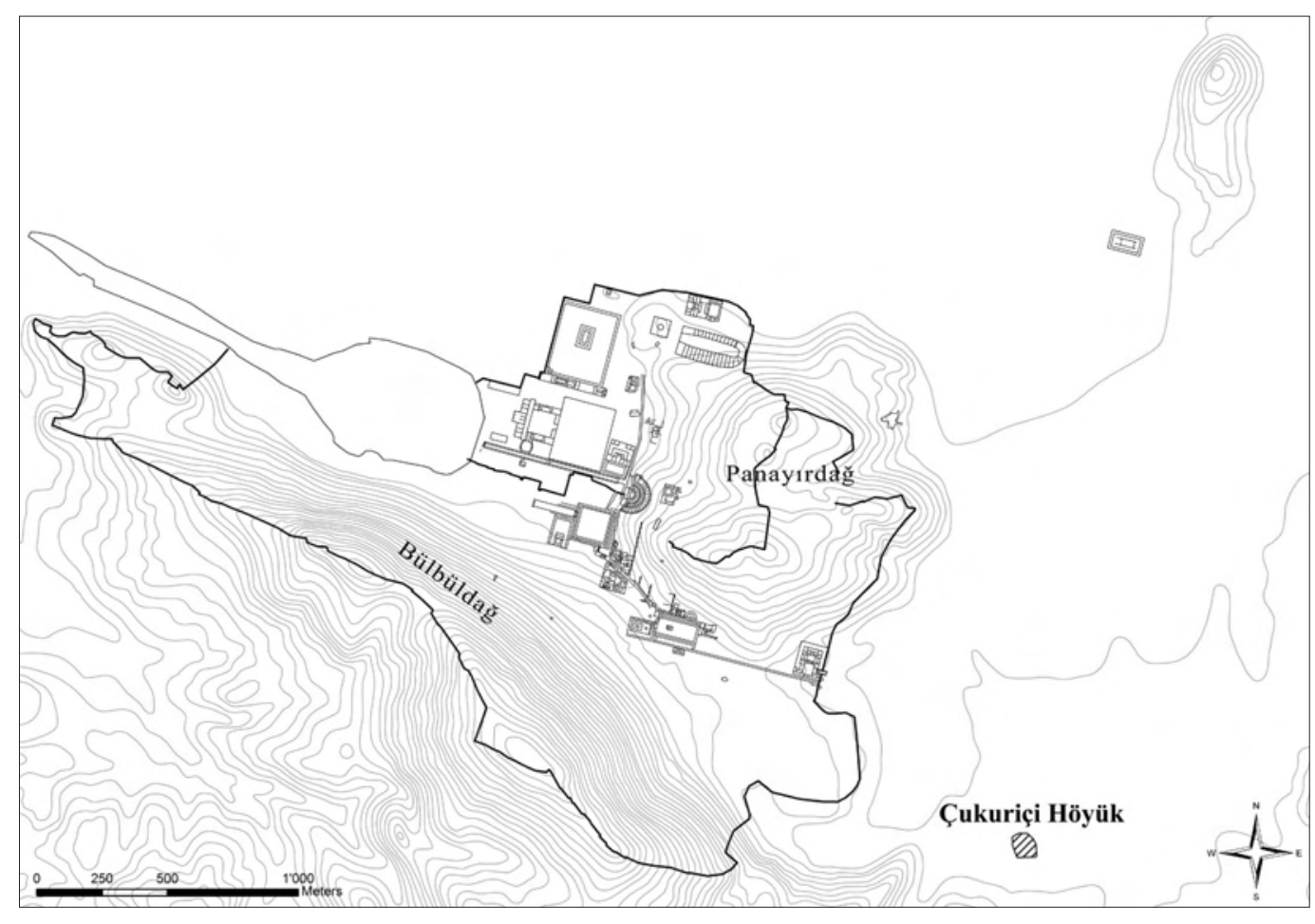

1 Lage des Çukuriçi Höyük im Raum von Ephesos

- An der Oberfläche und in unmittelbarer Umgebung des Hügels waren zunächst keine hellenistischen, römischen oder byzantinischen Architekturmerkmale zu erkennen, die postbronzezeitliche Störungen verursacht haben könnten. Eine ungestörte Schichtabfolge lag also im Bereich des Möglichen.

- Kurzfristige Rettungsgrabungen von einem Team des Ephesos Museums Selçuk 1995 erbachten in zwei kleinen Sondagen ein chronologisch breites Spektrum an Fundmaterial, das aber nicht stratigraphisch getrennt vorgelegt wurde ${ }^{7}$. Soweit dem Vorbericht entnommen werden kann, wurden nur die obersten 'Schichten ‘ abgebaut, konkrete Siedlungsreste gab es wohl nicht. Gerade der oberflächennahe Bereich scheint aber aus heutiger Sicht teilweise umgelagert, was die Zeitspanne des publizierten Materials vom Neolithikum/Chalkolithikum bis in die Frühbronzezeit erklären würde ${ }^{8}$.

- Schließlich war der Tell von unmittelbarer Zerstörung bedroht. Wie aus Luftaufnahmen der 1990er Jahre $^{9}$ und dem publizierten Plan der Rettungsgrabung ${ }^{10}$ zu schließen ist, war 2005 bereits mehr als die Hälfte des Hügels einplaniert worden. Der gesamte südliche Teil wurde offenbar freigegeben und in den Jahren nach 1995 bepflanzt. Auch die derzeitige Nordkante wurde vermutlich um mindestens $3 \mathrm{~m}$ abgegraben (Abb. 2). Durch die heutige Lage inmitten fruchtbarer Obstplantagen ist die unwiederbringliche Zerstörung des Tells in naher Zukunft abzusehen ${ }^{11}$.

Damit waren sowohl starke wissenschaftlich-archäologische Argumente als auch denkmalpflegerische Aspekte vorhanden, die ein systematisches Forschungsprojekt zum Çukuriçi Höyük rechtfertigten. Nach ersten vom ÖAI finanzierten Sondierungsgrabungen im Sommer $2006^{12}$ genehmigte schließlich der Fonds zur

\footnotetext{
${ }^{7}$ Evren - İçten 1998.

${ }^{8}$ Evren - İçten 1998, bes. Abb. 3-8.

9 Archiv ÖAI Wien: Infrarot-Orthophoto 1997; ÖAI, Institut für Photogrammetrie und Fernerkundung (TU Wien) und Abteilung für Photogrammetrie TU Istanbul (Bearbeitung 2001).

${ }^{10}$ Evren - İçten 1998, Abb. 2.

11 Zur Lage in den Plantagen s. Horejs 2008, Abb. 2-3.

${ }_{12}$ Auch hier gilt mein Dank dem ehemaligen Direktor des ÖAI, F. Krinzinger, für die Anschubfinanzierung des gesamten Unternehmens.
} 


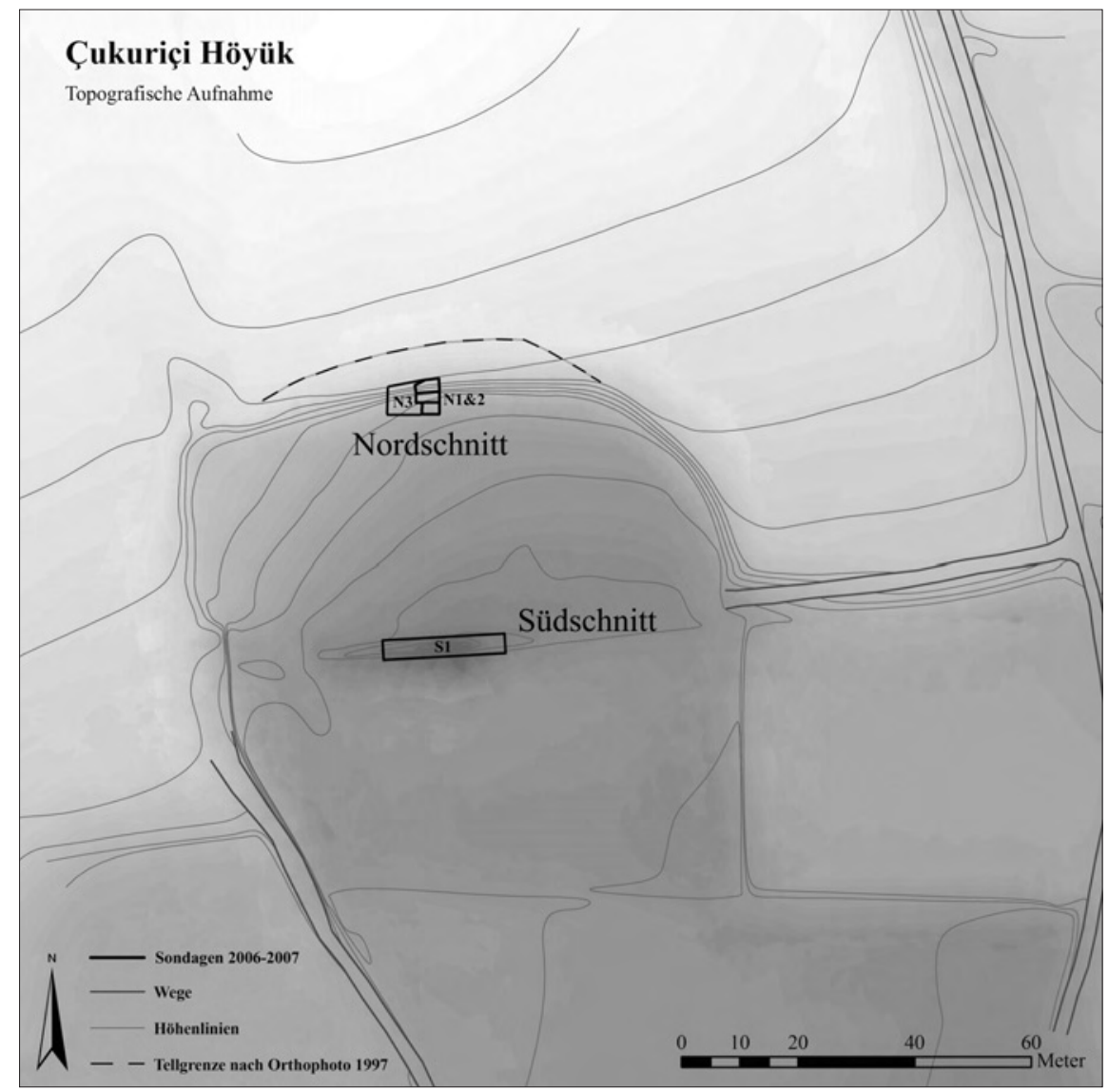

2 Topographischer Plan des Çukuriçi Höyük mit den Grabungsschnitten 2006/2007

Förderung der wissenschaftlichen Forschung ein dreijähriges Forschungsprojekt (Projektnr. P19859-G02) zum Thema »Grundlagenforschung zur Kupfer- und Bronzezeit in Ephesos« unter der Leitung der Autorin, in dessen Mittelpunkt die Untersuchung des Çukuriçi Höyük nun steht.

\section{Ziele und Methoden}

Da hier der erste Grabungsbericht dieses Projekts vorgelegt wird, sollen die wesentlichen Ziele und angewendeten Methoden kurz erläutert werden. Vorrangiges Ziel der gesamten Unternehmung ist zunächst die Erfassung des Fundplatzes in seinen primären archäologischen Aspekten; dazu zählen Funktion und Struktur sowie chronologische Tiefe und in weiterer Folge seine kulturhistorische Einbindung in die Mikroregion und schließlich auch in großräumige Fragestellungen. Dafür wird auf das gesamte Spektrum moderner archäologischer und interdisziplinärer Methoden zurückgegriffen ${ }^{13}$, wobei die Ausgrabung des Hügels selbst im Zentrum steht, die hier in Grundzügen beschrieben werden soll.

Die stratigraphische Grabung wird - leicht modifiziert - nach dem >Harris-Prinzip ${ }^{14}$ durchgeführt. Die direkte digitale Vermessung der photogrammetrisch dokumentierten Befunde ${ }^{15}$ erfolgt über einen Tachymat,

\footnotetext{
${ }^{13}$ Neben Archäobotanik und -zoologie, deren erste Ergebnisse hier kurz vorgestellt werden, gehören dazu auch Anthropologie, Geologie, Geophysik, Metallurgie, verschiedene Materialanalysen und Radiokarbonmessungen.

${ }^{14}$ Harris 1989.

${ }^{15}$ Der Terminus `Befund` wird hier inhaltlich gleichwertig zur unglücklichen deutschen Übersetzung der Harris’schen `Stratigraphical Unitく (SU bzw. SE [Stratigraphische Einheit]) verwendet.
} 


\section{Çukuriçi Höyük 2006}

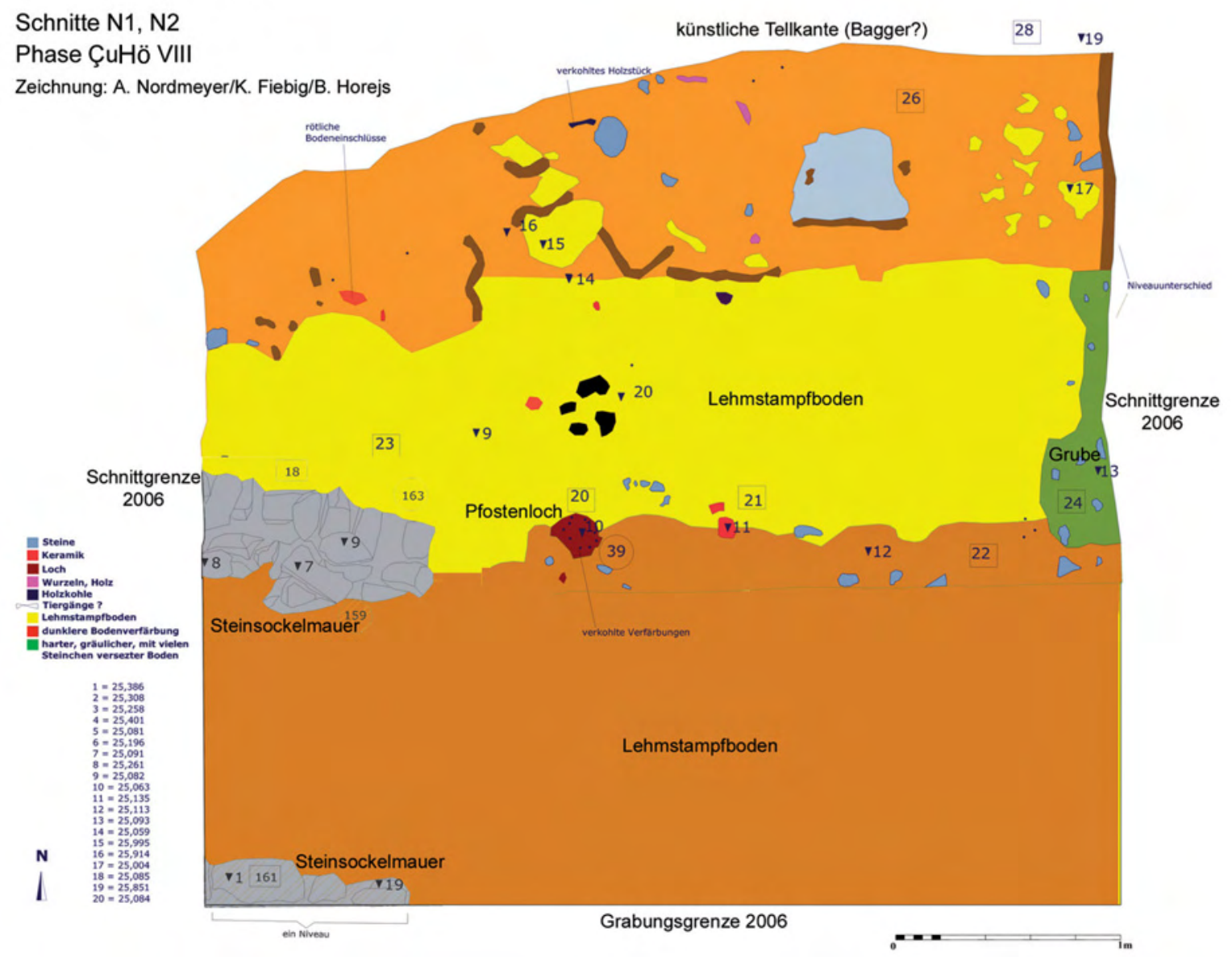

3 Aufsicht der Befunde aus Phase CuHö VIII

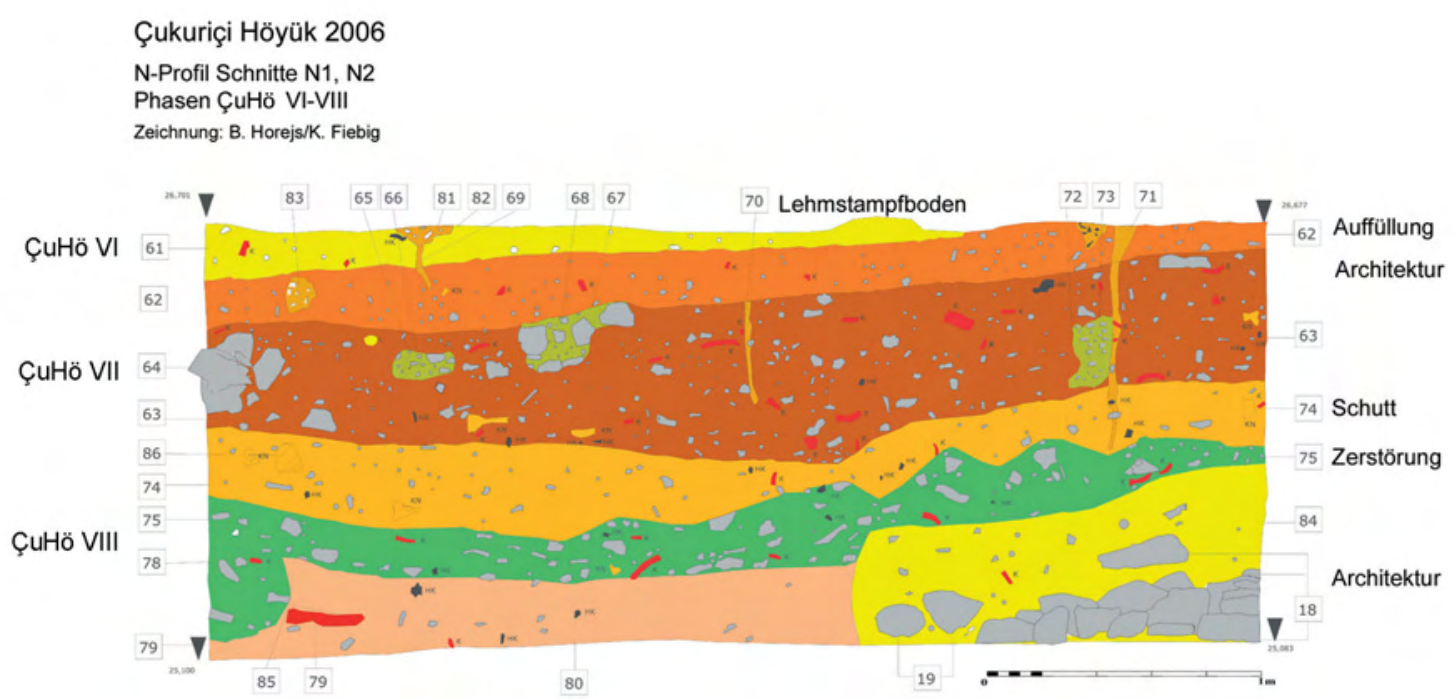

4 Nordprofil der Schnitte N1-N2 mit den Schichtabfolgen aus den Phasen ÇuHö VIII-VI 
der über eine Bluetooth-Verbindung mit einem speziellen feldtauglichen Notebook verbunden ist ${ }^{16}$. Dieses System erleichtert u. a. auch die Direkteinmessung von Funden in Bezug auf ihren stratigraphischen Kontext. Die bei Tellsiedlungen üblichen riesigen Mengen an Fundmaterial lassen sich auf diese Weise überschaubarer aufbereiten und elektronisch verwalten ${ }^{17}$.

Die weiteren geplanten oder bereits durchgeführten Untersuchungen der Anthropologie, Geologie, Geophysik, Metallurgie, die Methoden der verschiedenen Materialanalysen (z. B. NAA an Obsidian, Petrographie an Keramik) sowie die Radiokarbonmessungen werden erst nach abschließender Vorlage ihrer Ergebnisse vorgestellt und diskutiert werden. Im Folgenden werden die ersten Resultate der Sondierungsgrabung 2006 und der ersten regulären Kampagne 2007 zusammengefasst. Soweit sich bei derzeitigem Bearbeitungsstand gesicherte Aussagen treffen lassen, werden die ersten Erkenntnisse zu den oben formulierten Forschungszielen präsentiert. Einem Überblick über die Ergebnisse aus Botanik und Zoologie folgt ein abschließender Ausblick auf die weiteren Arbeiten.

\section{Grabungen 2006 und $2007^{18}$}

Mit unterschiedlicher Zielsetzung und Fragestellung wurde bislang in zwei Bereichen des Hügels, die im Folgenden als Nord- und Südschnitte bezeichnet werden (Abb. 2), gegraben. In ihrer Beschreibung sollen bloß einige wesentliche Befunde und Schichtabfolgen ausgewählter Siedlungsphasen vorgestellt werden, eine ausführliche Darstellung und Diskussion aller Kontexte bleibt der Publikation nach Abschluss der Grabungen vorbehalten.

\section{Nordschnitte (N1-N3)}

Die stratigraphisch tiefsten und chronologisch ältesten Bereiche wurden in den Schnitten N1-2 auf dem Niveau der heutigen Tellsohle erreicht. Diese Siedlungsphase (ÇuHö VIII) mit mehreren Nutzungshorizonten setzt sich aus verschiedenen Elementen zusammen (Abb. 3): Zwei annähernd parallele Steinsockelmauern aus grob behauenen und unbearbeiteten Steinen mit vermuteten Lehmplisseewänden stellen die Reste eines Hauses dar, dessen Größe und Form noch nicht näher zu definieren sind. Dazu gehört ein in der gesamten Schnittfläche erfasster mächtiger Lehmstampfboden, der verschiedene Ausbesserungen und Laufhorizonte aufweist und bis zur (vom Bagger?) künstlich geschaffenen Tellkante reicht. Vereinzelte Pfostenlöcher, eine Siedlungsgrube sowie ein breites Spektrum an Fundmaterial auf und in den einzelnen Nutzungsphasen erlauben eine erste Definition dieses Siedlungshorizontes. Über einer massiven Zerstörungsschicht, die diesen gesamten Kontext versiegelt, folgt eine mächtige Schuttablagerung (Abb. 4), die große Mengen an Keramik und Kleinfunden aller Kategorien enthielt.

Das signifikante Keramikspektrum des Siedlungshorizontes besteht aus überwiegend feinen orangefarbenen, roten und rotbraunen Waren mit teilweise fleckiger Oberfläche, die geglättet oder poliert sein kann ${ }^{19}$. Offene Gefäßformen mit sanftem S-Profil oder leicht geschwungener Wandung und leicht ausgezogener oder rund abschließender Lippe herrschen vor. Die Tunnelösen sind überwiegend vertikal, kurz und relativ breit, seltener schmal und lang gezogen (Abb. 5) ${ }^{20}$. Das Material aus der darüberliegenden Schuttschicht ist grundsätzlich sehr ähnlich, umfasst allerdings ein wesentlich breiteres Formen- und Warenspektrum.

\footnotetext{
${ }^{16}$ Ein auf ArcView basierendes Programm (Mobile Matrix) ermöglicht eine Direktverknüpfung der gemessenen Punkte mit einer auf Windows Access basierenden befundorientierten Datenbank.

${ }_{17}$ Zur Methode und Stratigraphie der Grabungen am Çukuriçi Höyük von 2006-2009 entsteht derzeit eine Diplomarbeit von M. Braun an der Universität Wien.

${ }^{18}$ Mein herzlicher Dank gilt hier allen studentischen Mitarbeiter/-innen beider Kampagnen: L. Apostel, M. Braun, K. Fiebig, Ch. Forstenpointner, S. Mattava, A. Nordmeyer, T. Sen-Öztürk, H. Öztürk, L. Toriser und J. Traumüller.

${ }^{19} \mathrm{Zu}$ der Definition der verwendeten deutschen Terminologie zur Oberflächenbehandlung an handgemachter Keramik s. Horejs 2007, 53-58.

${ }^{20} \mathrm{Zu}$ der Diskussion von Parallelen s. Horejs 2008, bes. Abb. 14.
} 

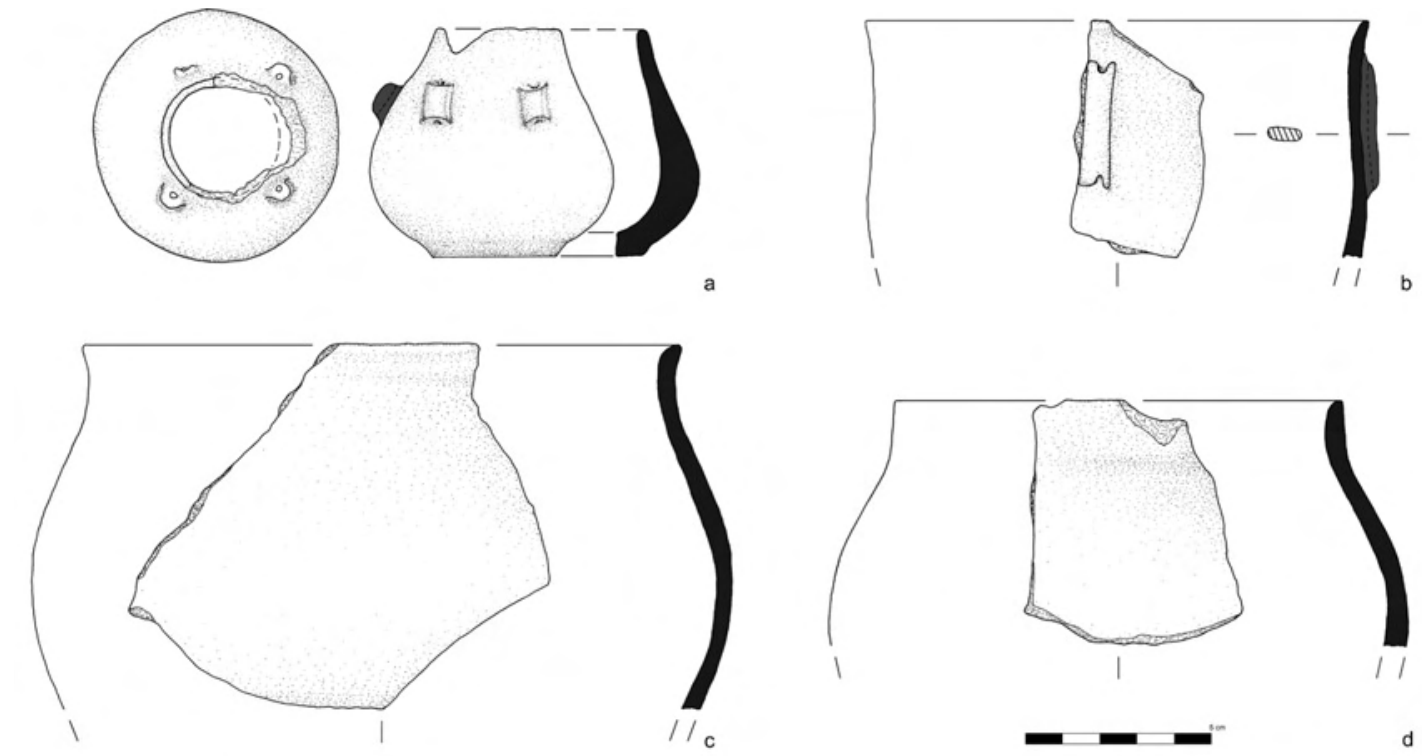

5 Monochrome geglättete Feinkeramik aus dem Lehmstampfboden der Phase ÇuHö VIII (Schnitt N2)

\section{Çukuriçi Höyük 2006 \\ Schnitt N1 \\ Phase ÇuHö VI \\ Zeichnung: A. Nordmeyer/K. Fiebig}

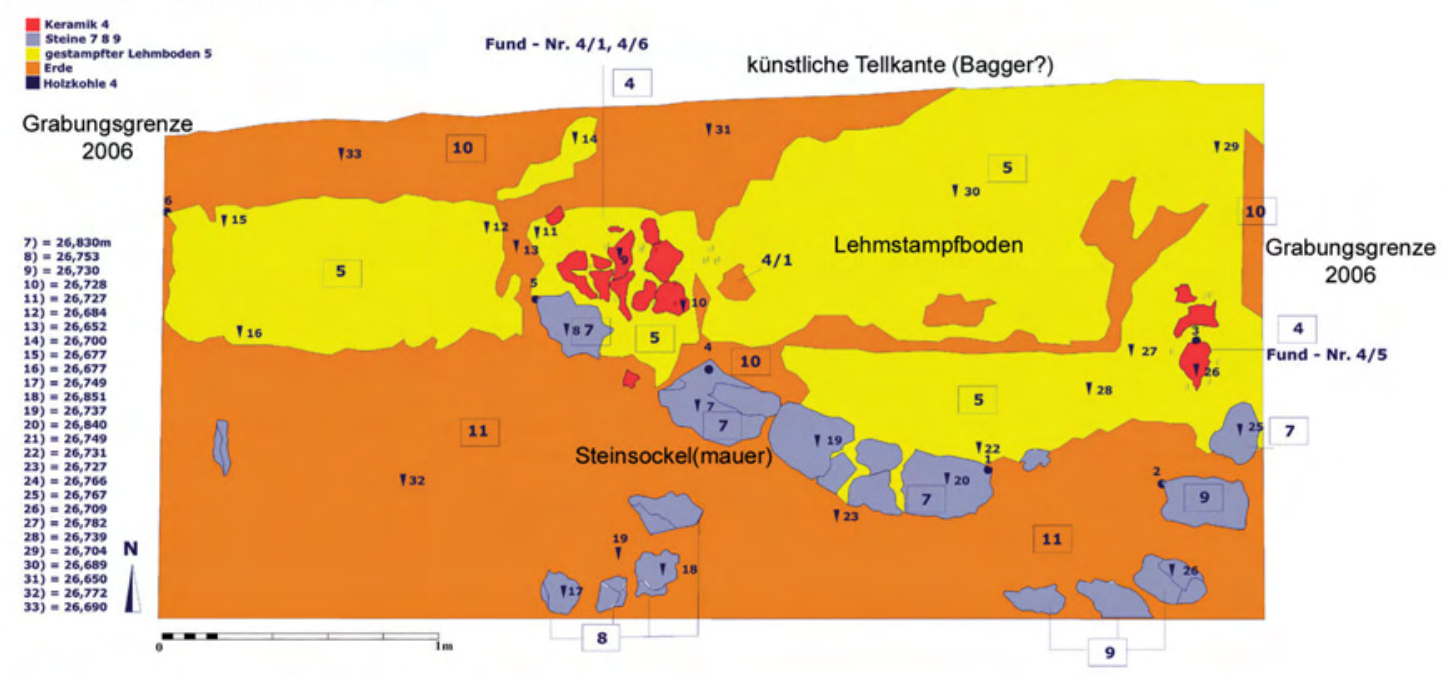

6 Aufsicht der Befunde aus Phase ÇuHö VI

Inwieweit dies für die chronologische Detailauswertung von Bedeutung ist, kann erst nach Abschluss der statistischen Auswertung entschieden werden ${ }^{21}$.

Eine erste vorläufige Beurteilung erlaubt eine Datierung dieser bislang ältesten Phase ÇuHö VIII in das späte Neolithikum oder frühe Chalkolithikum. Da dieser Zeithorizont an der anatolischen Westküste derzeit noch kaum erforscht ist und so gut wie keine stratigraphischen Abfolgen aus Siedlungen bekannt sind, ist

\footnotetext{
${ }^{21}$ Hier wird vor allem die Individuenanzahl der charakteristischen Scherben in Relation zum ausgegrabenen Schichtvolumen helfen. Zur Problematik und möglichen Lösung unterschiedlicher Schichtvolumina und Fundmaterialien aus Tellgrabungen s. Aslanis Hänsel (in Druck); Horejs 2007, 23.
} 


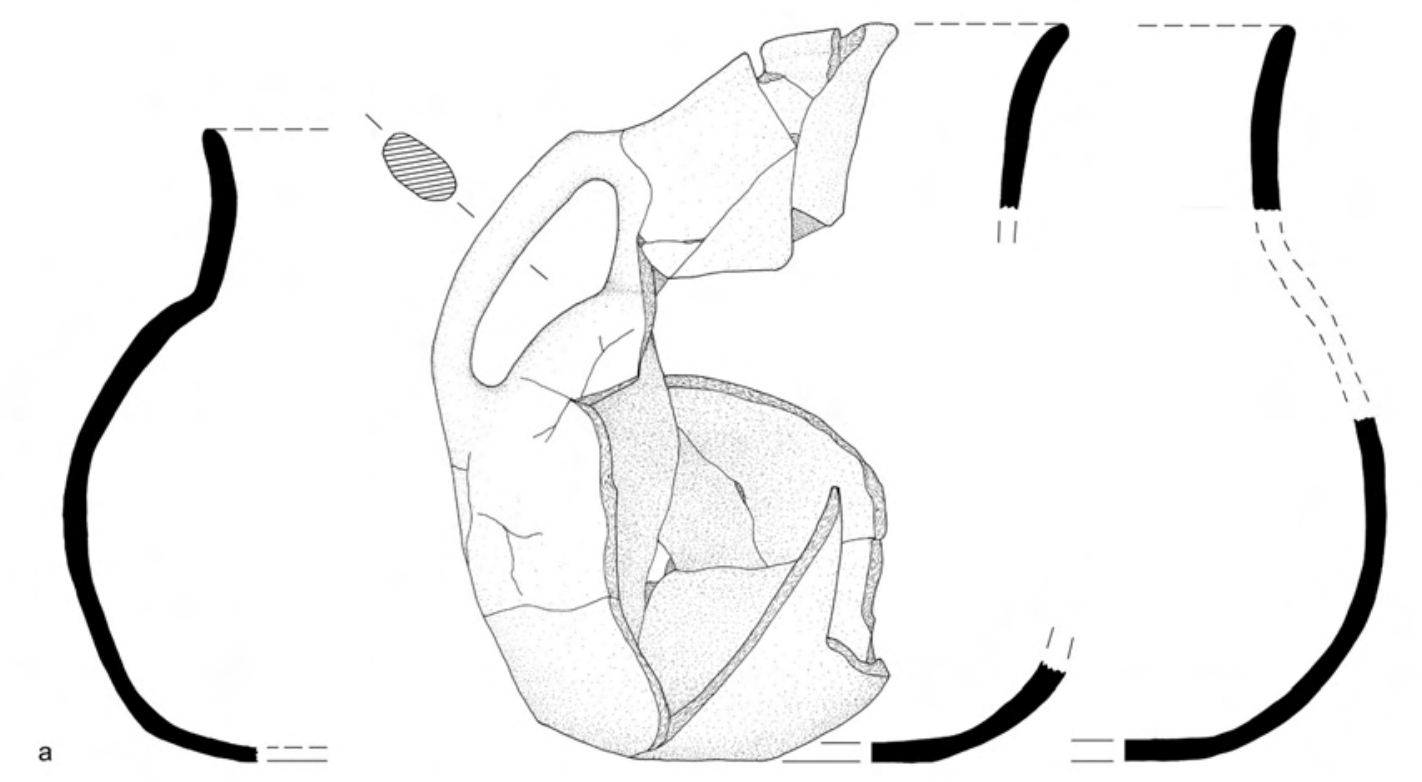

b
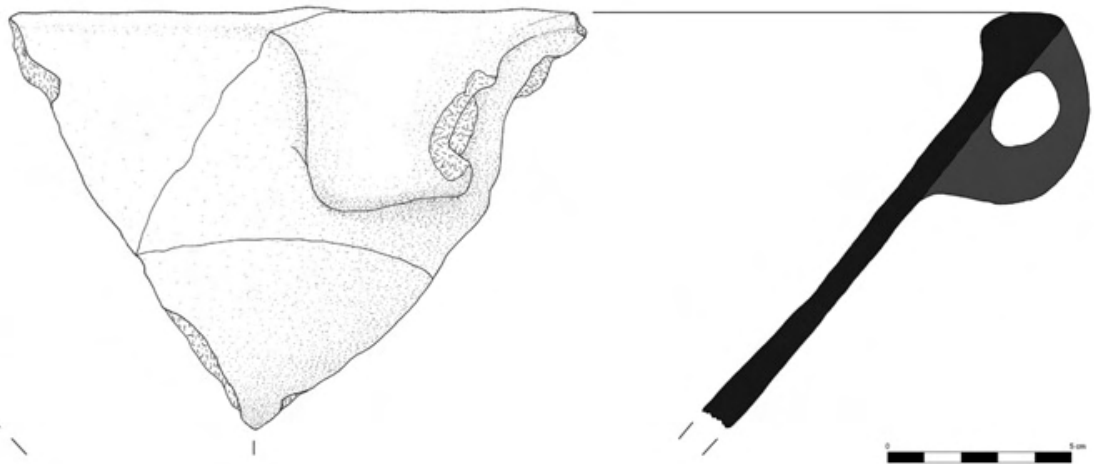

7 a Orangerot geglättete Feinkeramik aus Phase ÇuHö III (Schnitt S1); b Schwarz überzogene Feinware aus ÇuHö VI (Schnitt N1)

eine kulturhistorische Definition schwierig. In der derzeit laufenden Chronologiediskussion entspricht die Phase ÇuHö VIII etwa dem späten 7. oder frühen 6. Jahrtausend ${ }^{22}$.

Die darauffolgende Phase ÇuHö VII setzt sich wiederum aus mehreren Nutzungshorizonten zusammen, die dazugehörige Architektur ist allerdings weniger eindeutig zu definieren als in Phase VIII (Abb. 4). Zwischen zwei Fußbodenhorizonten in Schnitt N3, eindeutig innerhalb des Siedlungsverbandes, befand sich eine Kinderbestattung in einem Steinplattenkistengrab ${ }^{23}$. Die Definition eines mittleren oder späten Chalkolithikums ist in diesem Raum ebenso problematisch wie die der älteren Phasen, doch gibt es im Fundspektrum einige Indizien, die auf eine Datierung in das späte Chalkolithikum und damit das 4. Jahrtausend weisen.

Darauf folgt die davon klar abgegrenzte Siedlungsphase ÇuHö VI (Abb. 4. 6); die Reste einer gekrümmten Steinsockelmauer, teilweise ausgerissen, können vermutlich zu einem Oval- oder Apsidialbau ergänzt werden (Abb. 6). Ein massiver Lehmstampfboden mit Funden in Streu- und Sturzlage markiert den Nutzungshorizont im Innenbereich des Baus. Darunter sind u. a. schwarze gut geglättete und teilweise polierte Waren, wie die massiven Schalen mit innen verdicktem Rand und breitem, randständigem Bandhenkel (Abb. 7 b).

\footnotetext{
${ }^{22}$ Ausführliche Diskussion des Forschungsstandes bei Schoop 2005, 229-272; Chronologie nach Hauptmann - Özdoğan 2007; s. auch die Ergebnisse aus Ulucak (Çilingiroğlu u. a. 2004).

${ }^{23} \mathrm{Zu}$ Lage und Form des Grabes s. Horejs 2008, Abb. 8.
} 


\begin{tabular}{|ccc|}
\hline $\begin{array}{c}\text { Datierung } \\
\text { rezent }\end{array}$ & Südschnitt S1 & Nordschnitte N1-N3 \\
FBZ + 0 & I & \\
& III & II \\
FBZ I(-II) & $\begin{array}{c} \\
\text { Planierschichten } \\
\text { IV }\end{array}$ & \\
\hline nicht ausgegraben & V? & VI \\
(SpätCHA-) FBZ I & & Auffüllung \\
\hline SpätCHA (?) & & VII \\
\hline SpätNEO-FrühCHA & & VIII \\
\hline
\end{tabular}

8 Gliederung der Siedlungsphasen am Çukuriçi Höyük nach den Grabungen 2006/2007

Diese und andere signifikante Stücke legen eine Datierungsspanne vom Spätchalkolithikum bis in die frühe Frühbronzezeit nahe. Auch hier werden erst nach Abschluss einer statistischen Auswertung sowie einer größer ausgegrabenen Fläche exaktere Angaben möglich sein.

Im darüberliegenden und obersten Horizont der Nordschnitte sind die Umlagerungen durch rezente Baumgruben und anzunehmende Planierungen so stark, dass keine sicher geschlossenen Kontexte definiert werden konnten (Phase ÇuHö II), obwohl die Mehrheit des Fundspektrums chronologisch in die FBZ I zu weisen scheint.

Damit können in den Nordschnitten bislang drei sichere Siedlungsphasen (ÇuHö VIII-VI) und ein stark gestörter Horizont (ÇuHö II) festgestellt werden, die vom späten 7./frühen 6. bis in das 3. Jahrtausend v. Chr. reichen (Abb. 8).

\section{Südschnitt (S1)}

Bereits bei den ersten Begehungen und der topographischen Vermessung fiel ein rund $40 \mathrm{~m}$ langer, $2 \mathrm{~m}$ breiter und bis zu 2,5 m hoher >Schuttkegel an der heutigen Südgrenze des Hügels auf (Abb. 2). Nach den noch 2006 durchgeführten Reinigungsarbeiten zeichneten sich darin Befunde ab, die schließlich 2007 großflächig freigelegt wurden (Abb. 9). Es handelt sich um mindestens zwei größere und komplex angelegte Bauten aus massiven Steinsockelmauern mit Lehmziegel- oder Lehmwänden, deren Teile partiell in die einzelnen Räume gestürzt waren (Abb. 10). Durch den offensichtlich massiven rezenten Eingriff sowie starken Pflan-

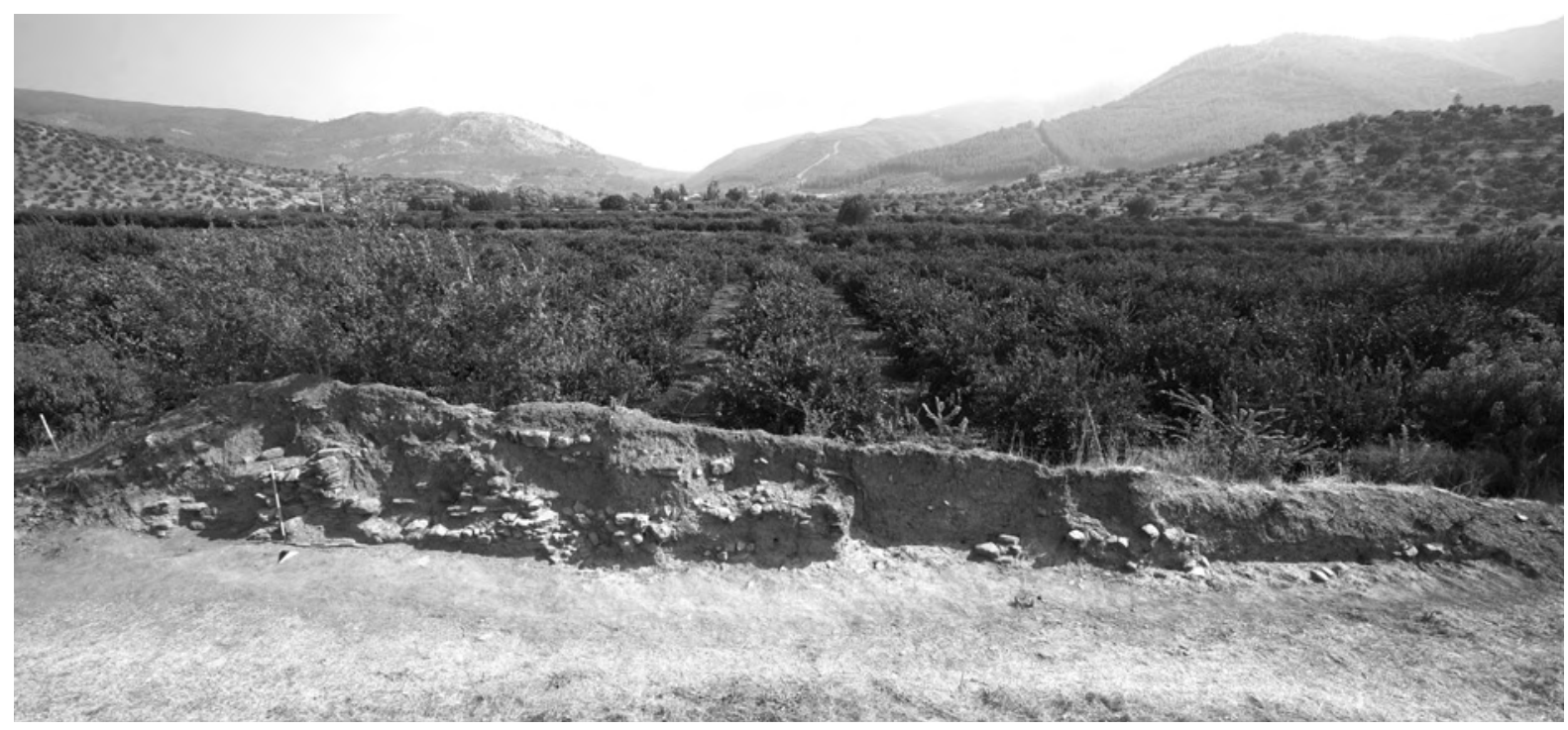

9 Südschnitt S1 (Südkegel) nach erster Reinigung und vor Grabungsbeginn 2006. Blickrichtung Süden 
zenbewuchs an allen Seiten des Kegels ist kein Raum vollständig erhalten geblieben (Abb. 10 a). Eine funktionale Definition einer Innen- und Außengliederung der Bauten ist daher nur vereinzelt möglich. Die im Folgenden verwendete Bezeichnung >Raum`steht demnach gleichermaßen auch für eventuell unüberdachte oder nicht komplett geschlossene Wohn- oder Nutzungseinheiten.

Der westliche Komplex besteht aus den Räumen 7-12 (Abb. 10 b. c) und stellt die stratigraphisch ältere Siedlungsphase in Schnitt S1 dar (ÇuHö IV). Die Mauern in diesem Bereich waren erstaunlicherweise bis zu zwölf Steinlagen in einer Höhe bis zu 0,90 m erhalten, wobei die eigentlichen Mauersohlen der ältesten Nutzungshorizonte bislang noch nicht erreicht wurden (Abb. 10 c). Die einzelnen Räume waren mit massiven Schuttschichten angefüllt, die sich vor allem aus den baueigenen Elementen und teilweise gut erhaltenen Funden zusammensetzten. Konkrete Hinweise auf den weiteren Wandaufbau waren in diesem Bereich nicht erhalten. Die dazugehörigen Lehmstampfböden setzen sich scheinbar Richtung Westen und Norden, direkt unter dem heutigen Laufhorizont des Hügels fort. Hier lassen die zukünftigen Grabungen auf weitere Befunde aus diesem Baukomplex hoffen.

Die nächst jüngere Siedlungsphase (ÇuHö III) ist durch teilweise massive Planierschichten von Phase ÇuHö IV getrennt. Es handelt sich um die Räume 1-6 im östlichen Bereich des Kegels (Abb. 10 b), die vermutlich zu einem größeren Komplex gehören. Die Konstruktion des Mauersockels entspricht der Bautechnik der Phase ÇuHö IV, mit dem entscheidenden Unterschied, dass sich im Schutt von Raum 1 nun auch Fragmente gebrochener und verbrannter Lehmziegel der aufgehenden Wandstruktur sowie Fragmente eines weißen Wandanstriches erhalten haben. Diese lagen zusammen mit einer großen Menge an Fundinventar auf dem jüngsten Nutzungshorizont in Form eines Lehmstampfbodens ohne jegliche Pflasterung (Abb. 10 e). Lage und Zustand der Funde belegen, dass es sich dabei um jene Objekte handelt, die sich bei der letzten Zerstörung des Gebäudes im Raum befunden haben müssen. Erstaunlich ist sowohl die große Anzahl als auch die Vielfalt des Materials: Einerseits handelt es sich um Gegenstände des alltäglichen Gebrauchs wie Dreifußkochtöpfe, Spinnwirtel, Schlag-, Klopf- und Reibsteine, Steinbeile, Knochenahle und Keramiklöffel, andererseits gehören auch bemalte und unbemalte Feinkeramik, wie Schnabelkannen (Abb. 7 a), sowie Perlen verschiedenen Materials, eine Nadel, Fragmente einer großen Kochplatte aus Ton und eine Mehrfachgussform zu dem Fundspektrum ${ }^{24}$. Neben der im gesamten Baukomplex massiven und variantenreichen Funddichte weisen auch noch weitere Elemente auf unterschiedlichste Tätigkeiten in diesem Siedlungsbereich hin, so beispielsweise die Öfen aus Lehmziegel in den Räumen 2 und 6 (Abb. 10 d).

Die Ausgrabung im Schnitt S1 ergab somit zwei weitere eindeutige Siedlungsphasen (ÇuHö IV-III), die jeweils aus mindestens einem größeren Gebäudekomplex mit mehreren Nutzungsphasen bestehen. Die noch nicht abgeschlossene Fundauswertung erlaubt eine erste vorläufige Datierung beider Phasen in ein eher frühes Stadium der frühen Bronzezeit (FBZ I-II).

\section{Fundplatzdefinition und Chronologie}

Die erste Auswertung der Grabungen 2006 und 2007 zeigt, dass es sich bei dem Çukuriçi Höyük um einen rechten< Tell handelt ${ }^{25}$, in dem bislang fünf Siedlungsphasen sicher definiert werden konnten (Abb. 8). Die noch hoch anstehenden Architekturreste der Phasen ÇuHö IV-III im Südschnitt waren auf den Bereich des >Schuttkegels« beschränkt, der gleichzeitig auch die Grenze zum nächsten Grundstücksbesitz Richtung Süden markiert. Da auch bei diesen Siedlungsphasen grundsätzlich von einer horizontalen Schichtablagerung auszugehen ist, muss der gesamte Bereich Richtung Norden und Süden abgetragen oder einplaniert worden sein. Die in den Nordschnitten festgestellten Baumgruben jüngster Pflanzungen liefern die entsprechende Erklärung für diesen Vorgang. Damit lässt sich festhalten, dass der Tell in den Jahren seit 1997 sowohl an allen Seiten als auch an seiner Oberfläche abgetragen und planiert wurde. Die ursprünglichen Ausmaße der Siedlungen lassen sich derzeit noch nicht definieren, können aufgrund aller Indizien aber nun als um einiges größer angenommen werden.

\footnotetext{
${ }^{24}$ Zur Gussform s. Horejs 2009.

${ }^{25}$ Definition nach Gogâltan 2005, 161.
} 

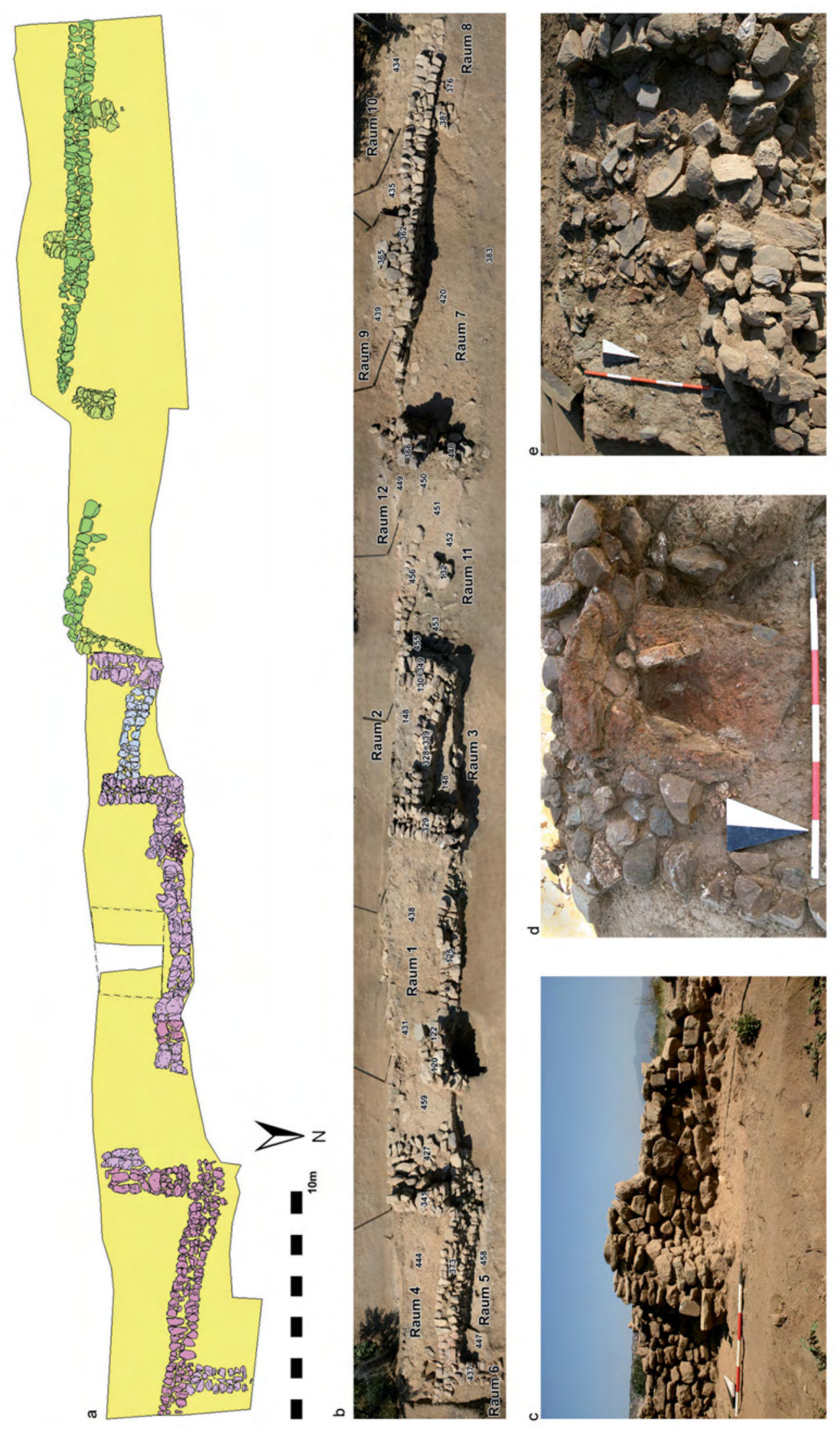

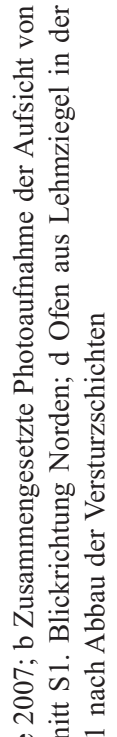

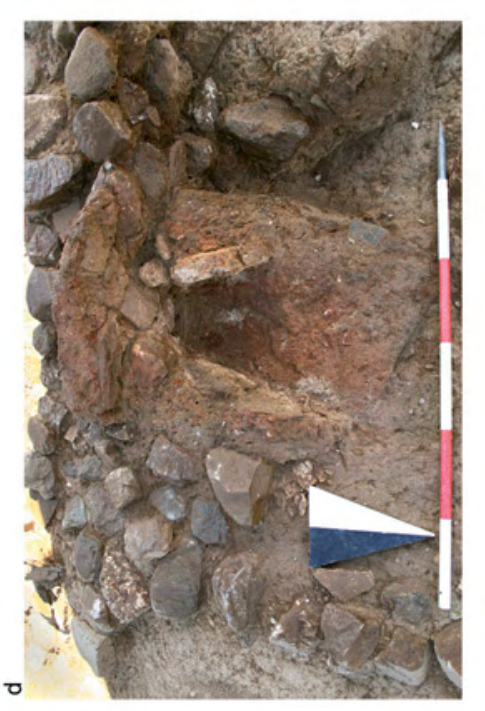

胥密的

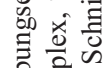

苟言:

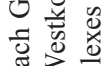

要

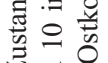

용

용

语言

证.

둥

ปั

ब

政

这

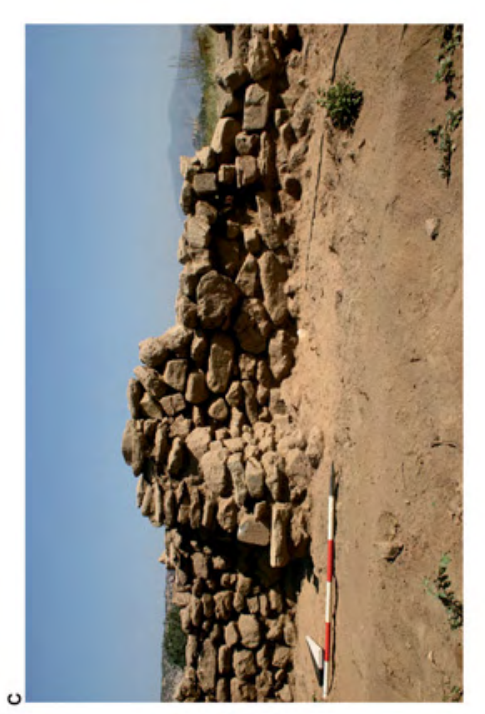

表荡

造

总总表

a 0

言容

识:

官 卷

疍品

言言言

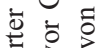

的范

焉青范

$ㅇ$ 
Eine erste vorläufige Datierung der Phasen zeigt folgendes Bild (Abb. 8): Die jüngsten Siedlungsphasen ÇuHö IV-III weisen in die FBZ I (-II?), die nächste sicher definierte Phase (ÇuHö VI) beinhaltet chronologisch ältere Elemente, die vom späten Chalkolithikum bis in die beginnende Frühbronzezeit reichen könnten. Darunter folgt mit Phase ÇuHö VII ein möglicher spätchalkolithischer Horizont, der wiederum auf der bislang ältesten Phase ÇuHö VIII liegt, die in das späte Neolithikum bis frühe Chalkolithikum datiert werden kann. Welche und wie viele Phasen noch darunter liegen, müssen zukünftige Arbeiten klären. Zusammenfassend gesagt, beinhaltet der Çukuriçi Höyük Siedlungsreste verschiedener Perioden, die nach den ersten Ergebnissen vom späten 7./frühen 6. Jahrtausend bis in das 3. Jahrtausend v. Chr. reichen.

Damit gehört der Tell nicht nur zu den bislang ältesten Fundorten in Westanatolien, sondern lässt aufgrund der fortgesetzten Nutzung in jüngeren Perioden auch auf neue Erkenntnisse zum 4. und 3. Jahrtausend hoffen. Der trotz der massiven rezenten Zerstörungen teilweise sehr gute Erhaltungszustand der Architektur sowie das in großen Mengen vorhandene breite Fundspektrum werden zudem wohl auch eine weiterführende Interpretation der Siedlungsfunktionen ermöglichen.

B. Horejs

\section{Erste Ergebnisse der zoologischen Analysen}

In den Grabungskampagnen 2006 und 2007 wurden am Çukuriçi Höyük bislang mehr als 1900 tierische Funde per Hand geborgen und ausgewertet; die Bearbeitung der bereits aussortierten Sedimente aus den Flotierproben steht noch aus. Der Großteil des tierischen Fundgutes akkumulierte als Haushaltsabfall des täglichen Lebens innerhalb der Siedlung. Rund 678 Funde stammen von oberflächlichen oder rezent gestörten Komplexen (ÇuHö I-II), die aufgrund ihrer wichtigen Funde ebenfalls aufgenommen und bearbeitet wurden. Da das keramische Fundmaterial aus diesen Horizonten überwiegend prähistorisch ist, können auch die Knochen, allerdings unter Vorbehalt, zur Interpretation herangezogen werden. 471 Reste stammen aus den Besiedlungsphasen der frühen Bronzezeit (ÇuHö III-IV). Aus dem Spätchalkolithikum (ÇuHö VII) liegen insgesamt 73, aus dem Frühchalkolithikum (ÇuHö VIII) 747 Tierreste vor.

Die Fleischversorgung erfolgte sowohl in der Frühbronzezeit wie auch im Chalkolithikum hauptsächlich durch Viehwirtschaft. Trotzdem scheint sich eine markante Änderung in der Viehzucht abzuzeichnen. Die wichtigsten Haustiere im Frühchalkolithikum sind kleine Hauswiederkäuer (Ovis aries, Capra hircus), Rinder (Bos primigenius f. taur.) und Schweine (Sus scrofa f. dom.), die neben vereinzelten Nachweisen von Hundeknochen (Canis lupus f. fam.) ausgeglichen verteilt scheinen. In der Frühbronzezeit hingegen überwiegen die kleinen Hauswiederkäuer deutlich gegenüber den anderen Arten.

Insgesamt beträgt der Anteil an Jagdwild im Frühchalkolithikum und der Frühbronzezeit nur rund $10 \%$ und bleibt im Spätchalkolithikum und der Phase ÇuHö I-II (’gestörte Fundkontexte ) unter $20 \%$. Hase (Lepus europaeus), Fuchs (Vulpes vulpes), Wildschwein (Sus scrofa) und Damwild (Dama dama) sind sowohl im Chalkolithikum als auch in der Frühbronzezeit belegbar. Funde vom Rothirsch (Cervus elaphus) und Auerochs (Bos primigenius) traten bislang nur in den frühchalkolithischen Befunden der Phase ÇuHö VIII auf. In frühbronzezeitlichen Kontexten konnten Reste von Bär (Ursus arctos) und Wolf (Canis lupus) geborgen werden. Frühbronzezeitlicher Vogelfang und Fischerei werden durch zwei Knochen kleiner entenartiger Vögel und das ossifizierte Wirbelzentrum eines Knorpelfisches aufgezeigt. Große und wildtierartig geformte Knochen >kleiner Wiederkäuer` deuten auf die Anwesenheit von Wildschaf (Ovis orientalis) und Wildziege (Capra aegagrus).

Die Häufung hauptsächlich mariner Mollusken weist offenbar auf eine nachhaltige Aufsammlung von Meeresweichtieren als Ergänzung zur Nahrung hin. Im Frühchalkolithikum sind die Napfschnecke (Patella sp.) und die Purpurschnecke (Hexaplex trunculus) nachweisbar. Die Frühbronzezeit erbrachte, abgesehen von den bereits erwähnten Arten, zahlreiche Gehäuse der Gemeinen Nadelschnecke (Gourmya vulgatum) und den Rest einer großen Helix-Landschnecke. Aus den Phasen ÇuHö I-II stammt der Nachweis für die Tonnenschnecke (Tonna galea). Die warzige Venusmuschel (Venus verrucosa) und die Miesmuschel (Mytilus galloprovincialis) finden sich nur im Frühchalkolithikum. Austernschalen (Ostrea edulis) sind in gerin- 
ger Zahl im Chalkolithikum und der Frühbronzezeit nachweisbar. Im Frühchalkolithikum überwiegen die Festsubstrate bewohnenden Muscheln, wie die Archenmuschel (Arca noe) und der Spondylus (Spondylus gaerderopus). Die essbare Herzmuschel (Cerastoderma glaucum) liegt nur in geringen Mengen vor. In der Frühbronzezeit aber dominieren diese Muscheln das Fundensemble der Mollusken.

Die Fundanteile von Rothirsch, Ur, Wildschwein, aber auch vom Hausschwein dürften ein Abnehmen der Bedeutung dieser Tiere in der Frühbronzezeit andeuten. Im selben Maß erhöht sich der Anteil des Damhirschs, vor allem in den Phasen ÇuHö I-II, und in der Viehwirtschaft werden Schafe und Ziegen wichtiger. In der Frühbronzezeit Südostanatoliens ${ }^{26}$ setzt sich mehrheitlich die Viehwirtschaft mit kleinen Hauswiederkäuern durch. Die Veränderung des Jagdwilds und die geänderte Strategie der Haustierzucht am Çukuriçi Höyük könnten auf eine Reduzierung des Waldbestandes im Verlauf vom Chalkolithikum bis zur Frühbronzezeit hinweisen ${ }^{27}$. Der Verlust der Bewaldung würde einen erhöhten Sedimenteintrag an der Küste bewirken, wodurch wiederum neue Lebensräume für im sandigen Flachwasser lebende Muscheln, wie die essbare Herzmuschel, entstünden. Die zu erwartenden Ergebnisse der künftigen Ausgrabungen verheißen jedenfalls neue Erkenntnisse für die Rekonstruktion der Kultur- und Umweltgeschichte des prähistorischen Siedlungsraumes des Çukuriçi Höyük.

\section{Erste Ergebnisse der botanischen Analysen}

Zur Bergung archäobotanischer Reste und von Kleinfauna wurden insgesamt 58 Bodenproben mit einem Gesamtvolumen von 5301 entnommen. Mit Ausnahme einer Probe aus der Phase ÇuHö VIII, die in das Chalkolithikum datiert, stammen alle Proben aus der frühen Bronzezeit ${ }^{28}$. Die Extraktion der Reste erfolgte mittels einer herkömmlichen Flotationsanlage ${ }^{29}$. Dabei konnten 965 Pflanzenreste geborgen werden, was einer durchschnittlichen Dichte von knapp zwei Pflanzenresten pro Liter Erdreich entspricht. Botanische Reste liegen in zwei Erhaltungsformen vor: Verkohlt und mineralisiert, wobei die mineralisierten Reste nur ca. $5 \%$ des gesamten Materials ausmachen und vorwiegend aus Feigenkernen bestehen.

Die verkohlten Pflanzenreste lassen sich in fünf große Gruppen einteilen: Getreide, Hülsenfrüchtler, Ölund Faserpflanzen, Obstgehölze und krautige Wildpflanzen. Beim Getreide liegen ausschließlich Körner vor; Spreu, also Spelzen oder Spindelfragmente, konnten bisher nicht nachgewiesen werden. Die Körner sind schlecht erhalten und stark fragmentiert, was sich im hohen Prozentsatz der nicht bestimmbaren Exemplare niederschlägt. Die bestimmbaren Getreidekörner entfallen fast zur Gänze auf Gerste (Hordeum vulgare); Einkorn (Triticum monococcum) und Emmer (T. dicoccum) sind hingegen nur schwach vertreten $(29: 2: 1)$. Gerste hat eine kürzere Vegetationsdauer als Weizen und kommt deshalb besser mit marginalen Bedingungen wie salzhaltigen oder nährstoffarmen Böden und Wassermangel zurecht. $\mathrm{Ob}$ sie deshalb bevorzugt angebaut wurde, oder ob es sich um eine kulturelle Präferenz handelt, müssen weitere Forschungen klären.

Hülsenfrüchtler sind mit mehreren Arten vertreten, wobei allerdings nur die Linse (Lens culinaris) als eindeutiges Nahrungsmittel eingestuft werden kann. Alle anderen Arten, wie beispielsweise die Linsenwicke

\footnotetext{
${ }^{26}$ M. Frangipane und G. Siracusano diskutieren die Veränderungen der Ernährungsstrategien im 3. und 4. Jtsd. anhand der Fundstellen von Arslantepe, Horum Höyük, Hassek Höyük und Kurban. In der Frühbronzezeit setzte sich im südöstlichen Anatolien bereits überwiegend die Wirtschaft mit kleinen Hauswiederkäuern durch, wohingegen sich im Spätchalkolithikum noch Unterschiede in den Fundquantitäten von Schwein und kleinen Hauswiederkäuern manifestieren (Frangipane - Siracusano 1998, 273; Bartosiewicz 2005,154-160).

${ }^{27}$ Bottema u. a. 2001, 347 beschreibt anhand von Pollenkurven für den Bereich östlich des Maramarameers eine ähnliche Entwicklung der Vegetation, die schließlich im beinahe gänzlichen Verschwinden des Laubwaldes an der Grenze vom Spätchalkolithikum zur Frühbronzezeit gipfelt.

${ }^{28}$ Erst mit Beginn der systematischen Grabungen 2007, wo besonders die Phasen der Frühbronzezeit untersucht wurden, konnte eine Flotationsanlage eingesetzt werden. Die in der Sondierungsgrabung 2006 freigelegten chalkolithischen Horizonte wurden hingegen noch nicht geschlemmt.

${ }^{29}$ s. z. B. Schneider - Kronberger 1991.
} 
(Vicia ervilia), sind Bestandteil der Vegetation der Umgebung, wachsen auch als Ackerunkräuter, könnten als Viehfutter genutzt oder auch zur menschlichen Ernährung angebaut worden sein.

Öl- bzw. Faserpflanzen sind nur durch wenige Leinsamen (Linum usitatissimum) für das Chalkolithikum belegt; für die frühe Bronzezeit konnten sie bisher nicht nachgewiesen werden.

Erstaunlich hoch ist die Anzahl der Samen- bzw. Fruchtreste von Obstgehölzen. Feige (Ficus carica), Weintraube (Vitis vinifera), Mandel (Amygdalus sp.) und Olive (Olea europaea) stellen ca. $35 \%$ des verkohlten Pflanzenmaterials. Während des Chalkolithikums und der frühen Bronzezeit waren Wildformen von allen aus dem Çukuriçi Höyük nachgewiesenen Obstarten im östlichen Mittelmeerraum weit verbreitet; die Früchte wurden sicherlich gesammelt und vermutlich gab es auch schon sehr früh Bestrebungen zur Kultivierung dieser regelmäßig genutzten Pflanzen. Frühbronzezeitliche Wein- und Feigenkerne sind beispielsweise aus Kastanas ${ }^{30}$ in Makedonien bekannt, in Troia ${ }^{31}$ fanden sich aus demselben Zeitraum außerdem Olivenkerne. Fragmente von Mandelschalen, die bereits dem späten Neolithikum/frühen Chalkolithikum (ÇuHö VIII) zuzuordnen sind, wurden in Haçılar ${ }^{32}$ nachgewiesen.

Bei den wildwachsenden Pflanzen dominieren Schmetterlingsblütler (Fabaceae) und Gräser (Poaceae). Beide Familien stellen wichtige Komponenten der lokalen Vegetation dar, zu ihnen zählen häufige Ackerunkräuter, aber auch Pflanzen, die gerne als Viehfutter genutzt werden.

U. Thanheiser

\section{Zusammenfassung und Ausblick}

Nach den Ausgrabungen 2006 und 2007 lassen sich bereits erste konkrete und neue Erkenntnisse zum Çukuriçi Höyük formulieren. Es handelt sich bei diesem Fundplatz zweifelsfrei um einen Tell, der mindestens fünf Siedlungsphasen enthält, die vom späten 7./frühen 6. bis zum 3. Jahrtausend v. Chr. datieren. Architektur und Bautechnik ändern sich im Lauf der Perioden, konstant bleiben einige Details, wie ungepflasterte Lehmstampfböden und Steinsockel als Wandunterbau. Nachdem die chronologische Tiefe nun grob erfasst werden konnte, werden die nächsten Ausgrabungen vor allem die Erfassung flächiger Siedlungsstrukturen in ihrer zeitlichen Abfolge zum Ziel haben. Bei den jüngsten Phasen beginnend, werden zunächst die frühbronzezeitlichen Siedlungsreste im Mittelpunkt stehen.

Das Fundmaterial spiegelt ein breites Spektrum an Gattungen wider, dessen Parallelen vorläufig vom südwestanatolischen Seengebiet über Zentralanatolien bis zu den Regionen des Marmarameers und der westanatolischen Küste reichen. Rein ägäische Elemente lassen sich bislang kaum feststellen. Deutlich ist bereits die lokale Produktion verschiedenster Objekte, deren Rohstoffe (wie Obsidian) aber eingetauscht werden mussten. Einige Indizien weisen neben der normalen Nutzung auch auf die Herstellung von Metallobjekten, deren Zusammensetzung und Produktionsverfahren noch untersucht werden müssen. Inwieweit dazu auch konkrete Werkstattareale erhalten sind, werden die nächsten Ausgrabungen zeigen.

Die Untersuchungen der tierischen und pflanzlichen Reste geben einen ersten Einblick in das zukünftige Potenzial des Hügels zu den Fragen nach Ressourcennutzung und Landschaftsentwicklung im Wandel der Jahrtausende. Während die Bewohner des Çukuriçi Höyük im Chalkolithikum eine vielfältige Viehwirtschaft betreiben, spezialisieren sie sich im 3. Jahrtausend vorwiegend auf die kleinen Hauswiederkäuer. Jagdwild scheint hingegen in allen Perioden keine große Bedeutung für die Nahrungsbeschaffung besessen zu haben. Deutlich ist bislang die Dominanz der besonders widerstandsfähigen Gerste als Nahrungsgrundlage. Die Frage der Kultivierung der nachgewiesenen Feigen, Weintrauben, Mandeln und Oliven bleibt zukünftigen Analysen vorbehalten.

Die Veränderung im Molluskenbestand und die Zunahme von Damhirsch lassen erste mögliche Rückschlüsse auf einen Wandel des Naturraumes bis zur Frühbronzezeit zu. Diese und zukünftige Erkenntnisse

\footnotetext{
30 Kroll 1983, 149.

31 Riehl 1999, 105.

32 Helbaek 1970.
} 
könnten zu der derzeit laufenden Diskussion einer möglichen Klimaveränderung im späten 7. Jahrtausend und damit einhergehenden Landschaftsveränderungen und Küstenlinienverschiebungen wichtige Beiträge liefern.

Die Ausgrabungen von 2006 und 2007 am Çukuriçi Höyük geben einen ersten Einblick in einen in Ephesos bislang kaum erforschten Zeitraum. Der Tell und sein unmittelbares Umfeld bergen die Voraussetzungen für eine erste grundlegende kulturhistorische Definition dieser Mikroregion vor dem Hintergrund einer sich im Lauf der Jahrtausende vom beginnenden Chalkolithikum bis in die Frühbronzezeit permanent verändernden Landschaft. Umso wünschenswerter ist es, diesen Fundort zu sichern, weitere Zerstörungen zu verhindern und ihn den interessierten Besuchern zugänglich zu machen.

B. Horejs

\begin{abstract}
Abgekürzt zitierte Literatur
Aslanis - Hänsel (in Druck) I. Aslanis - B. Hänsel, Das prähistorische Olynth. Ausgrabungen in der Toumba Agios Mamas 1994-1996. Die Grabung und der Baubefund, PAS (Rahden/Westfalen, in Druck).

Bartosiewicz 2005

L. Bartosiewicz, Animal remains from the excavations of Horum Höyük, Southeast Anatolia Turkey, in: H. Buitenhuis - A. M. Choyke - L. Martin - L. Bartosiewicz - M. Mashkour, Archaeozoology of the Near East VI, Archaeological Research and Consultancy Publicaties 123 (Groningen 2005) 150-162.

Bottema u. a. 2001 S. Bottema $-\mathrm{H}$. Woldring $-\dot{I}$. Kayan, The late quaternary vegetation history of Western Turkey, in J. J. Roodenberg - L. C. Thissen, The Ilıpınar Excavations 2 (Leiden 2001) 327-354.

Çevik 2002

Ö. Çevik, Prehistorik Çağlarda Kuşadası ve Çevresi, in: A. G. Şerifoğlu (Hrsg.), Geçmişten Geleceğe Kuşadası. Sempozyum 23-26 Şubat 2000 (Izmir 2002) 71-79.

Çilingiroğlu u. a. 2004 A. Çilingiroğlu u. a., Ulucak Höyük. Excavations conducted between 1995 and 2002 (Louvain 2004).

Cutting 2005

M. V. Cutting, The Neolithic and Early Chalcolithic Farmers of Central and Southwest Anatolia. Household, community and the changing use of space, BAR IntSer 1435 (Oxford 2005).

DAI Jahresbericht 2006 Jahresbericht des Deutschen Archäologischen Instituts 2006, Abteilung Istanbul, Didyma, AA 2007/2, $126-128$.

Evren 1999

A. Evren, Efes Çukuriçi Höyüğü. 1996 yıl kazısı, Arkeoloji ve Sanat 92, 1999, 22-30.

Evren - İçten 1998

A. Evren - C. İçten, Efes Çukuriçi ve Arvalya (Gül Hanım) Höyükleri, in: Müze Kurtarma Kazıları Semineri 8 (Ankara 1998) 111-133.

Frangipane - Siracusano 1998 M. Frangipane - G. Siracusano, Changes in subsistence strategies in East Anatolia during the 4th and 3th millenium BC, in: P. Anreiter - L. Bartosiewicz - E. Jerem - W. Meid, Man and the Animal World. Studies in memoriam Sandor Bökönyi, Archaeolingua 8 (Budapest 1998) 237-246.

French 1997 D. H. French, Early Bronze Age Pottery in Western Anatolia. A Summary 1970-1995, in: Ch. Dou-

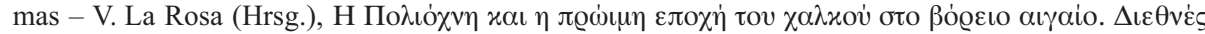

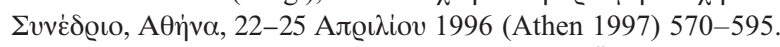

Friesinger - Krinzinger 1999 H. Friesinger - F. Krinzinger (Hrsg.), 100 Jahre Österreichische Forschungen in Ephesos. Akten des Symposions Wien 1995, DenkschrWien 260 = AForsch 1 (Wien 1999).

Gogâltan 2005 F. Gogâltan, Der Beginn der bronzezeitlichen Tellsiedlungen im Karpatenbecken: chronologische Probleme, in: B. Horejs - R. Jung - E. Kaiser - B. Teržan (Hrsg.), Interpretationsraum Bronzezeit. Bernhard Hänsel von seinen Schülern gewidmet, Universitätsforschungen zur Prähistorischen Archäologie 121 (Berlin 2005) 161-179.

Groh 2006 St. Groh unter Mitarbeit von V. Lindinger - K. Löcker - W. Neubauer - S. S. Seren, Neue Forschungen zur Stadtplanung in Ephesos, ÖJh 75, 2006, 47-116.

Harris 1989 E. C. Harris, Principles of Archaeological Stratigraphy (London 1989).

Hauptmann - Özdoğan 2007 H. Hauptmann - M. Özdoğan, Die Neolithische Revolution in Anatolien, in: Die ältesten Monumente der Menschheit. Ausstellungskatalog Karlsruhe 2007 (Stuttgart 2007) 26-36.

Helbaek 1970 H. Helbaek, The plant husbandry of Hacilar, in: J. Mellaart (Hrsg.), Excavations at Hacilar 1 (Edinburgh 1970) 189-244.

Horejs 2007 B. Horejs, Das prähistorische Olynth 3. Die handgemachte spätbronzezeitliche Keramik der Schichten 13 bis 1, PAS 21 (Rahden/Westfalen 2007).

Horejs 2008 B. Horejs, Çukuriçi Höyük. A New Excavation Project in the Eastern Aegean. Aegeo-Balkan Prehistory $<$ http://www.aegeobalkanprehistory.net/article.php?id_art=9>

Horejs 2009 B. Horejs, Metalworkers at the Çukuriçi Höyük? An Early Bronze Age Mould and a >Near Eastern weight from Western Anatolia, in: T. L. Kienlin - B. Roberts (Hrsg.), Metals and Societies. Studies in honour of Barbara S. Ottoway, Universitätsforschungen zur prähistorischen Archäologie 169 (Bonn 2009) 358-368.
\end{abstract}


Kouka 2002

Kroll 1983

Lichter 2005

Lloyd - Mellart 1962

Meriç 2003

Meriç 2007

Niemeier 2007

Özdoğan 2007

Özdoğan - Başgelen 1999

Parzinger 1993

Riehl 1999

Scherrer 2001
Meriç - Mountjoy 2002

O. Kouka, Siedlungsorganisation in der Nord- und Ostägäis während der Frühbronzezeit (3. Jt. v. Chr.), Internationale Archäologie 58 (Rahden/Westfalen 2002).

H. Kroll, Kastanas. Ausgrabungen in einem Siedlungshügel der Bronze- und Eisenzeit Makedoniens 1975-1979. Die Pflanzenfunde, PAS 2 (Berlin 1983).

C. Lichter (Hrsg.), How Did Farming Reach Europe? Anatolian-European Relations from the Second Half of the $7^{\text {th }}$ through the first half of the $6^{\text {th }}$ Millenium Cal BC. Proceedings of the International Workshop Istanbul, 20-22 May 2004, Byzas 2 (Istanbul 2005).

S. Lloyd - J. Mellart, Beycesultan I. The Chalcolithic and Early Bronze Age Levels. Occasional Publications of the British Institute of Archaeology at Ankara 6 (London 1962).

R. Meriç - P. Mountjoy, Mycenaean Pottery from Bademgediği Tepe (Puranda) in Ionia: A preliminary report, IstMitt 52, 2002, 79-98.

R. Meriç, Excavations at Bademgediği Tepe (Puranda) 1999-2002: A Preliminary Report, IstMitt 53, 2003, 79-98.

R. Meriç, Ein Vorbericht über eine spätbronzezeitliche befestigte Höhensiedlung bei Metropolis in Ionien: Die Arzawa-Stadt Puranda?, in: J. Cobet u. a. (Hrsg.), Frühes Ionien. Eine Bestandsaufnahme, Panionion-Symposion Güzelçaml 26. September - 1. Oktober 1999, MilForsch 5 (Mainz 2007) 27-36.

W.-D. Niemeier, Milet von den Anfängen menschlicher Besiedlung bis zur ionischen Wanderung, in: J. Cobet u. a. (Hrsg.), Frühes Ionien. Eine Bestandsaufnahme, Panionion-Symposion Güzelçamlı 26. September - 1. Oktober 1999, MilForsch 5 (Mainz 2007) 3-20.

M. Özdoğan, Amidst Mesopotamia-centric and Euro-centric approaches: the changing role of the Anatolian peninsula between the East and the West, AnSt 57, 2007, 17-24.

M. Özdoğan - N. Başgelen (Hrsg.), Neolithic in Turkey. The Cradle of Civilisation (Istanbul 1999).

H. Parzinger, Studien zur Chronologie und Kulturgeschichte der Jungstein-, Kupfer- und Frühbronzezeit zwischen den Karpaten und Mittlerem Taurus, RGF 52 (Mainz 1993).

S. Riehl, Bronze Age Environment and Economy in the Troad. The Archaeobotany of Kumtepe and Troy, BioArchaeologica 2 (Tübingen 1999).

P. Scherrer, The historical topography of Ephesos, in: D. Parrish (Hrsg.), Urbanism in Western Asia Minor. New Studies on Aphrodisias, Ephesos, Hierapolis, Pergamon, Perge and Xanthos, JRA Suppl 45 (Portsmouth 2001) 58-93.

Schneider - Kronberger 1991 M. Schneider - W. Kronberger, Die Flotation archäobotanischer Proben, AÖ 2/1, 63-64.

Schoop 2005

U.-D. Schoop, Das anatolische Chalkolithikum. Eine chronologische Untersuchung zur vorbronzezeitlichen Kultursequenz im nördlichen Zentralanatolien und den angrenzenden Gebieten, Urgeschichtliche Studien 1 (Großschönau 2005).

Yakar 1985 268 (Oxford 1985).

Mag. Dr. Barbara Horejs

\% Österreichisches Archäologisches Institut, Franz Klein-Gasse 1, A-1190 Wien

E-Mail: barbara.horejs@oeai.at

\section{Dr. Alfred Galik}

Arbeitsgruppe für Archäozoologie und Vergleichende Morphologie, Abteilung für Pathobiologie, Institut für Anatomie, Veterinärmedizinische Universität Wien, Veterinärplatz 1, A-1210 Wien

E-Mail:alfred.galik@vu-wien.ac.at

Dr. Ursula Thanheiser

VIAS - Archäobotanik, UZA II, Althanstraße 14, A-1090 Wien

E-Mail: ursula.thanheiser@univie.ac.at

Abbildungsnachweis: Abb. 1: Plangrundlage: Ch. Kurtze, (C) ÖAI; Abb. 2: Ch. Kurzte, (C) ÖAI; Abb. 3. 6: Aufnahme A. Nordmeyer, Digitalisierung K. Fiebig/B. Horejs, (C) ÖAI; Abb. 4: Aufnahme B. Horejs, Digitalisierung K. Fiebig/B. Horejs, (C) ÖAI; Abb. 5: a. ÇuHö 06/165/02; b. ÇuHö 06/26/01/34; c. ÇuHö 06/23/01/01; d. ÇuHö 06/26/01/09, Zeich- 
nungen S. Mattova/J. Traumüller, C ÖAI; Abb. 7: a. ÇuHö 06/196/02; b. ÇuHö 06/04/05/01, Zeichnungen S. Mattova/ J. Traumüller, (C) ÖAI; Abb. 8: B. Horejs, C ÖAI; Abb. 9: Photo N. Gail, C ÖAI (A-W-OAI-ECU-00679); Abb. 10: a. Plangrundlage: M. Braun, (C) ÖAI; b. Bearbeitung: M. Braun/N. Gail/B. Horejs, C) ÖAI; c. Photo: M. Braun, C ÖAI (A-W-OAI-ECU-02035); d. Photo: L. Toriser, (C) ÖAI (A-W-OAI-ECU-01636); e. Photo: F. Krinzinger, (C) ÖAI (A-WOAI-ECU-01251). 